\title{
DNA Variant in the RPGRIP1L Gene Influences Alternative Splicing
}

\author{
Emma Reble ${ }^{a-c}$ Yu Fenga, b Karen G. Wigg ${ }^{a, b}$ Cathy L. Barr ${ }^{a-e}$ \\ ${ }^{a}$ Genetics and Development Division, Krembil Research Institute, University Health Network, Toronto, ON, Canada; \\ ${ }^{b}$ Program in Neurosciences and Mental Health, The Hospital for Sick Children, Toronto, ON, Canada; ' Institute of

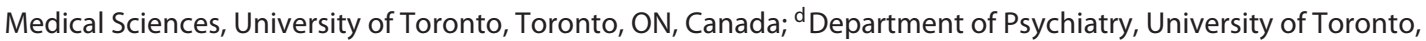 \\ Toronto, ON, Canada; ${ }^{\text {eDepartment }}$ of Physiology, University of Toronto, Toronto, ON, Canada
}

\author{
Keywords \\ RPGRIP1L · Joubert syndrome - Meckel syndrome · Bipolar \\ disorder - Schizophrenia - Autism . Gene regulation . \\ Splicing · Transcription · Gene expression · Genetics . \\ Genome-wide association study
}

\begin{abstract}
The retinitis pigmentosa GTPase regulator interacting protein 1-like (RPGRIP1L) gene encodes a ciliary protein that is critical for processes related to brain development, including development of left-right asymmetry, sonic hedgehog signaling, and neural tube formation. RPGRIP1L is a risk factor for retinal degeneration, and rare, deleterious variants in the RPGRIP1L gene cause Joubert syndrome and Meckel syndrome, both autosomal recessive disorders. These syndromes are characterized by dysfunctional primary cilia that result in abnormal development - and even lethality in the case of Meckel syndrome. Genetic studies have also implicated RPGRIP1L in psychiatric disorders by suggestive findings from genome-wide association studies and findings from rare-variant exome analyses for bipolar disorder and de novo mutations in autism. In this study we identify a common variant in RPGRIP1L, rs7203525, that influences alternative splicing, increasing the inclusion of exon 20 of RPGRIP1L.
\end{abstract}

We detected this alternative splicing association in human postmortem brain tissue samples and, using a minigene assay combined with in vitro mutagenesis, confirmed that the alternative splicing is attributable to the alleles of this variant. The predominate RPGRIP1L isoform expressed in adult brains does not contain exon 20; thus, a shift to include this exon may impact brain function.

(c) 2019 S. Karger AG, Basel

\section{Introduction}

Retinitis pigmentosa GTPase regulator interacting protein 1-like (RPGRIP1L) encodes for a protein that localizes to the basal bodies and transition zone of primary cilia $[1,2]$. RPGRIP $1 L$ is critical for proper primary cilium function, as knockout mice have a ciliopathy phenotype and die at birth [1,3]. Vierkotten et al. [1] showed that RPGRIP1L is necessary for multiple processes during embryonic development, including left-right asymmetry development, neural tube formation, and cilium-related sonic hedgehog signaling.

Variants affecting RPGRIP1L have been linked to multiple diseases including Joubert syndrome, Meckel syndrome, $\mathrm{COACH}$ (cerebellar vermis aplasia, oligophrenia,

\section{KARGER}

(c) 2019 S. Karger AG, Basel

karger@karger.com

www.karger.com/mnp
Dr. Cathy L. Barr

Krembil Research Institute

60 Leonard Avenue, Krembil Discovery Tower 8KD-412

Toronto, ON M5T 0S8 (Canada)

E-Mail Cathy.Barr@uhnresearch.ca 
congenital ataxia, coloboma, and hepatic fibrosis) syndrome, nephronophthisis, and retinal degeneration [2]. Joubert syndrome and Meckel syndrome, both ciliopathies [3], are both autosomal recessive disorders and are thought to represent a spectrum of the same disorder, with Meckel syndrome being on the extreme end of the spectrum, as it is lethal and is characterized by renal cystic dysplasia, polydactyly, and central nervous system malformation. Joubert syndrome is a severe but nonlethal condition, often diagnosed by the "molar tooth sign" brain malformation phenotype visible on magnetic resonance imaging [4]. Other core hallmarks of Joubert syndrome include ataxia, hypotonia, and intellectual disability [4]. Abnormal eye movement and function, cognitive impairment, development delay, polydactyly, and renal and liver diseases have also been reported as features of the disorder [4]. Individuals with Joubert and Meckel syndromes are at risk for retinal degeneration, and rare [5] and common variants in RPGRIP1L have been implicated in retinal degeneration [6].

Primary cilia are essential integrators and conveyors of signal transduction and are critical for multiple aspects of brain development and function including cortical development, neural progenitor development, early neuronal connectivity, and neuronal migration and differentiation $[7,8]$. Knockdown experiments in the murine developing cortex implicate the murine homologue of RPGRIPIL in neuronal migration and, consequently, correct placement of neurons in the developing cortex [7]. Given these key functions in brain development, primary ciliary function in general $[9,10]$, and $R P G R I P 1 L$ specifically, have been implicated in neuropsychiatric disorders. Injecting NMDAR antagonists, psychotomimetics often used to model psychiatric phenotypes, into rat and mouse brains causes a decrease in primary cilium length and downregulates RPGRIP1L mRNA expression, among other primary cilium-related genes [11]. Further, in multiple genome-wide association studies (GWAS), markers in $R P G R I P 1 L$ were identified as suggestive of schizophrenia and bipolar disorder $[12,13]$, with the most significant $p$ values of $\sim 10^{-7}$ for bipolar disorder [14]. RPGRIP1L was also identified by rare-variant exome analyses for bipolar disorder [15] and de novo mutations in autism [16]. Further supporting RPGRIPIL as a risk factor for psychiatric disorders are findings of a higher rate of neuropsychological and behavioral symptoms among patients with Joubert syndrome [17]. RPGRIP1L has also been found to physically interact in cells with NIMA-related kinase 4 (NEK4), a gene associated with psychiatric disorders [13], at the basal bodies of the cilia and is thought to act as a scaffolding protein to recruit proteins to the primary cilia [18]. Schizophrenia-associated single nucleotide variants (SNVs) were shown to be associated with differentially spliced NEK4 transcripts in the CommonMind Consortium data set [19].

Because of the key role of RPGRIP1L in neural cell function and brain development, we sought to identify functional variants in RPGRIP1L affecting gene and protein expression as possible contributors to disease. Given that the majority of the risks for complex traits are thought to alter gene expression including splicing [20], we mined data sets of predicted splicing variants to identify SNVs predicted by [21], or correlated with [22, 23], alternative splice isoforms and identified rs8050354 as significantly correlated with alternative splicing of exon 20 (position hg19 chr16:53,672,222-53,672,323; exon 20 as annotated in RefSeq, and exon 22 in GTEx). This variant was associated with bipolar disorder and schizophrenia at $p=$ $4.48 \times 10^{-5}$ in a recent joint analysis [24]. We showed that alterative splicing of exon 20 was correlated with this SNV in adult human brain tissues. Using minigene assays combined with in vitro mutagenesis, we identify rs7203525, an SNV in high linkage disequilibrium (LD) with rs8050354, to be responsible for the alternative splicing in SH-SY5Y cells. The predominate RPGRIP1L isoform expressed in adult brains in the GTEx database does not contain exon 20; thus, a shift to include this exon may impact brain function.

\section{Methods}

Human Brain Tissue Samples

The aim of this study was to identify alternative splicing changes associated with disease risk. Because the risk SNVs analyzed in this study are common in the population, brain samples from controls carry the risk alleles, and therefore were used to study the relationship between these variants and alternative splicing. Brain tissue samples from 7 schizophrenia patients, 4 bipolar patients, and 79 controls were obtained from the Harvard Brain Tissue Resource Center, the Douglas-Bell Canada Brain Bank, and the Eunice Kennedy Shriver National Institute of Child Health and Human Development (Table 1).

As the purpose of this study was to investigate the effect of common risk variants on alternative splicing, all analyses were performed with the samples separated by genotype regardless of the disease status. The control postmortem brain tissue samples were from healthy individuals $<65$ years of age with no neurological or psychiatric disorder. The postmortem interval for these tissues was $<24 \mathrm{~h}$. The exclusion criteria for the control brain tissue samples included individuals where a neurological disease may have influenced the tissue quality, such as stroke, prolonged hypoxic death, Alzheimer's disease, or other psychiatric or neurological disorders. The gender, age, ethnicity, and cause of death were provided for
98

Mol Neuropsychiatry 2019;5(suppl 1):97-105 DOI: $10.1159 / 000502199$
Reble/Feng/Wigg/Barr 
each individual. Two brain regions relevant to psychiatric disease were analyzed for this study: (1) dorsolateral prefrontal cortex (DLPFC) Brodmann area 9 and (2) the hippocampus.

\section{Splicing SNV Identification}

We mined the splicing prediction and correlation data sets included the SPIDEX database, three data sets from GTEx (Altrans, sQTLseekeR, and protein-truncating variants), and a data set of DLPFC sQTLs identified in the CommonMind Consortium data $[21,23,25-27]$. These splicing data sets consist of variants associated with a splicing change based on RNA-Seq and genotype data (GTEx and DLPFC sQTLs) or of variants that are predicted by a computational model to cause a splicing change based on 1,393 features from the two flanking introns and exons of the target exon (SPIDEX) [21]. Identified SNVs were mined for their relationship to associated markers for psychiatric disease $[13,24]$.

The predicted RPGRIP1L alternative splicing variants identified were then validated in a genotyped panel of human brain tissue samples. RPGRIP1L transcript isoform expression was tested based on the splicing data set predictions using DLPFC and hippocampal cDNA from the human brain tissue samples. Primers were designed flanking the alternatively spliced exon of RPGRIP1L in order to size-separate the PCR-amplified fragments by gel electrophoresis (forward primer: 5'-TCGTGGATATCATGCCACATCA-3'; reverse primer: 5'-GCTTTGTTCTGCAAGCTGAC- $\left.3^{\prime}\right)$. Each PCR reaction consisted of $4 \mathrm{ng}$ of $\mathrm{cDNA}, 2 \mathrm{X}$ PCR Master Mix (Thermo Fisher Scientific, Waltham, MA, USA), and $0.5 \mu \mathrm{M}$ of the primers in exons 19 and 21 of RPGRIP1L. An initial denaturation was performed at $95^{\circ} \mathrm{C}$ for $3 \mathrm{~min}$, followed by 40 cycles consisting of a 30 -s denaturation at $95^{\circ} \mathrm{C}$, a 30 -s anneal at $55^{\circ} \mathrm{C}$, and a 30 -s extension at $72^{\circ} \mathrm{C}$. A final extension step was performed at $72{ }^{\circ} \mathrm{C}$ for $10 \mathrm{~min}$. The PCR amplification was then run on a $2 \%$ agarose gel, and the presence of splicing isoforms was identified based on size separation of amplified fragments, with the RPGRIP1L transcript containing exon 20 amplifying a 239-bp fragment and the RPGRIP1L transcript skipping exon 20 amplifying a 137-bp fragment. All identified alternative transcripts were gel purified and sequenced.

Upon identification of the alternatively spliced isoform, a quantitative digital droplet PCR (ddPCR) assay was designed in order to quantify the proportion of each splicing isoform in each sample. Probe-based assays were designed, with one assay amplifying the RPGRIP1L isoform with the exon spliced in using a HEX fluorescent dye for the probe, and a second assay amplifying the exon spliced out using a FAM fluorescent dye for the probe (forward primer: 5'-AGCTGACCTTCAGATAGTAAAGAC-3'; reverse primer: 5'-AGAAGGTATCTTTCGTGGATATCAT-3'; probe for full length: $5^{\prime}$-TGGGAACATGTGGAACAGTCAGCA-3'; probe for exon skipping: $5^{\prime}$-ACTGCCTTCTTGTGAAACACTCTGATGT- $3^{\prime}$ ). Each ddPCR reaction consisted of $10 \mathrm{ng}$ of cDNA, 2X ddPCR Supermix for Probes (No dUTP) (Bio-Rad Laboratories, Hercules, CA, USA), 20X FAM probe mix, and 20X HEX probe mix. An initial denaturation was performed at $95^{\circ} \mathrm{C}$ for 10 min, followed by 40 cycles consisting of a 30 -s denaturation at $95^{\circ} \mathrm{C}$ and a 1 -min combined anneal and extension step at $58.5^{\circ} \mathrm{C}$. A deactivation step was performed at $98^{\circ} \mathrm{C}$ for $10 \mathrm{~min}$, followed by a hold at $4^{\circ} \mathrm{C}$. These assays were run as duplex ddPCRs on each brain sample. Each droplet with either the full length or the exonskipped transcript, determined by either FAM or HEX fluorescence, was then counted. The fractional abundance "FA" of the
Table 1. Demographics of the individuals from whom the brain sample tissues were collected

\begin{tabular}{llll}
\hline & Schizophrenia & $\begin{array}{l}\text { Bipolar } \\
\text { disorder }\end{array}$ & Control \\
\hline Samples, $n$ & 7 & 4 & 79 \\
\hline Gender & 7 male & 3 male & 50 male \\
& 0 female & 1 female & 29 female \\
\hline Mean age, years & 42.3 & 44.25 & 40.2 \\
\hline Ethnicity & 7 Caucasian & 4 Caucasian & $\begin{array}{l}69 \text { Caucasian } \\
9 \text { African American } \\
\text { mixed ethnicity }\end{array}$ \\
\hline
\end{tabular}

exon-skipped transcript compared to the total number of transcripts amplified was determined by the following equation: $\mathrm{FA}=$ $\mathrm{A} /(\mathrm{A}+\mathrm{B})$. "A" refers to the copies per microliter of the alternatively spliced transcript, in this case the exon-skipped transcript, and " $\mathrm{B}$ " refers to the copies per microliter of the full-length transcript, in this case the transcript with the exon spliced in.

The fractional abundance was determined for each individual brain sample and was then run as an ANOVA with sex, RNA integrity number (RIN), age, ethnicity, postmortem interval, and genotype of the candidate SNVs as factors. Only samples with an RIN $>6.1$ were used in this analysis (mean $7.41 \pm 0.67$, range 6.19.2). Sex, RIN, age, ethnicity, and postmortem interval were not significantly related to the alternative isoform and were not included in the further analyses. The fractional abundance of each sample compared to their genotype was also plotted as a boxplot using "plotly" in R. For each plot, the upper and lower hinges are the 3rd and 1st quartiles, respectively, and the upper and lower whiskers are the largest and smallest values contained within 1.5 times the interquartile range. Outliers are values that extend above or below 1.5 times the interquartile range.

\section{SNV Genotyping}

DNA from the brain tissue samples was genotyped using the Illumina OmniExpress Platform ( 713,599 SNVs genotyped). PLINK version 1.9 was used for quality control and analyses (http://pngu.mgh.harvard.edu/purcell/plink/) [28]. SNVs were removed if they deviated from Hardy-Weinberg equilibrium $(p<$ 0.0001 ), had a minor allele frequency $<0.05$, or had a call rate $<98 \%$. Ethnicity of the samples was checked using principal component analysis. The genotypes were imputed using the Michigan Imputation Server (https://imputationserver.sph.umich.edu/index.html) using the Haplotype Reference Consortium (HRC version r1.1 2016) [29]. SNPs with a low imputation score $\left(r^{2}<0.3\right)$ were removed and a final round of quality control was performed. Approximately 5.3 million SNPs remained after imputation and quality control. For the SNVs used in this study, rs8050354 was genotyped ( $\mathrm{T}$ minor allele frequency 0.314 ) and rs7203525 was imputed (T minor allele frequency 0.289, imputation $r^{2}=0.99876$ ).

RNA Extraction from Brain Tissue Samples and SH-SY5Y Transfected Cells

RNA was extracted using TRIzol reagent and the PureLink RNA Mini Kit following the manufacturer's protocol, with the ad- 
ditional DNAse step added to ensure removal of genomic DNA (Invitrogen, Carlsbad, CA, USA). Eighty-nine DLPFC and 65 hippocampal brain samples had high-quality RNA and were used to make cDNA. cDNA was synthesized using the ImProm-II Reverse Transcriptase protocol (Promega, Madison, WI, USA). For cDNA synthesis of RNA from transfected cells, the RTRHC R primer $\left(5^{\prime}\right.$-GCTTTGCAGCAACAGTAAC-3') specific to the RHCglo plasmid was used instead of oligo(dT) in order to synthesize cDNA specific to the transcribed exons in from the plasmid, and not the endogenous cDNA.

\section{Minigene Assays}

A minigene assay was designed to test the candidate SNVs significantly associated with the alternative splicing change. The region surrounding the alternatively spliced exon 20 as well as the candidate SNV was cloned into the SalI and XbaI restriction sites of the RHCglo splicing vector using forward and reverse primers in introns 19 and 20 of RPGRIP1L (forward primer: $5^{\prime}$-ATATATGTCGACACTCAGAGATACTGTTTGGATG-3'; reverse primer: $5^{\prime}$-ATATATTCTAGAGAGACTCAGAAAACAACCACTT-3') [30]. Both clones, either containing the major or the minor allele of the candidate SNV, were confirmed by sequencing.

Clones with either allele of the candidate SNV were transfected into SH-SY5Y cells separately according to the manufacturer's protocol (Lonza Group, Basel, Switzerland). RNA was extracted from the cells $24 \mathrm{~h}$ after transfection. RT-PCR was performed using a reverse primer specific to the RHCglo plasmid as the RT primer, and reverse and forward primers specific to the RHCglo plasmid for the PCR (forward primer: $5^{\prime}$-CATTCACCACATTGGTGTG-3'; reverse primer: $5^{\prime}$-GCTTTGCAGCAACAGTAAC- $\left.3^{\prime}\right)$. Each PCR reaction consisted of $4 \mathrm{ng}$ of cDNA, 2X PCR Master Mix (Thermo Fisher Scientific), and $0.5 \mu \mathrm{M}$ of the forward and reverse primers. An initial denaturation was performed at $95^{\circ} \mathrm{C}$ for $3 \mathrm{~min}$, followed by 40 cycles consisting of a 30 -s denaturation at $95^{\circ} \mathrm{C}$, a 30 -s anneal at $55^{\circ} \mathrm{C}$, and a 30 -s extension at $72^{\circ} \mathrm{C}$. A final extension step was performed at $72^{\circ} \mathrm{C}$ for $10 \mathrm{~min}$. The PCR amplification was then run on a $2 \%$ agarose gel, and the presence of splicing isoforms was identified based on size separation of amplified fragments, with the transcript containing exon 20 at $290 \mathrm{bp}$ and the transcript skipping exon 20 at $188 \mathrm{bp}$. All identified alternative transcripts were gel purified and sequenced.

Site-directed mutagenesis was performed according to the manufacturer's protocol (Q5 ${ }^{\circledR}$ Site-Directed Mutagenesis Kit; New England Biolabs, Ipswich, MA, USA). Forward primers containing the nucleotide change for the substitution of the risk by the non-risk allele (or vice versa) were used (forward major allele primer: 5'-ATAAAAAATGACATACATGC-3'; forward minor allele primer: $5^{\prime}$-ATAAAAAATAACATACATGC-3'; reverse primer: $5^{\prime}$-TTATTACTAAATCCCAAATCATT- $3^{\prime}$ ). Plasmids with the risk allele were changed to the non-risk allele and vice versa, and the sequence changes were confirmed by sequencing. The plasmids were then transfected separately into SH-SY5Y cells and tested for their effect on alternative splicing using PCR primers designed for the exons of the RHCglo vector as described above.

Quantification of the cDNA from the minigene assays was performed using the Bioanalyzer High Sensitivity DNA Analysis assay. A PCR was performed with forward and reverse primers specific to the RHCglo plasmid (forward primer: $5^{\prime}$-CATTCACCACATTGGTGTG-3'; reverse primer: $5^{\prime}$-GCTTTGCAGCAACA-
GTAAC- $3^{\prime}$ ). Each PCR reaction consisted of $4 \mathrm{ng}$ of cDNA, 2X PCR Master Mix (Thermo Fisher Scientific), and $0.5 \mu \mathrm{M}$ of the forward and reverse primers. An initial denaturation was performed at $95^{\circ} \mathrm{C}$ for $3 \mathrm{~min}$, followed by 35 cycles consisting of a 30 -s denaturation at $95^{\circ} \mathrm{C}$, a 30 -s anneal at $55^{\circ} \mathrm{C}$, and a 30 -s extension at $72^{\circ} \mathrm{C}$. A final extension step was performed at $72^{\circ} \mathrm{C}$ for $10 \mathrm{~min}$. The PCR amplification was then run on the DNA Bioanalyzer, and the concentration of the peaks from the fragment with exon 20 spliced in as well as the fragment with exon 20 spliced out was determined. The percent spliced in "PSI" of exon 20 was determined by the following equation: $\mathrm{PSI}=\mathrm{B} /(\mathrm{A}+\mathrm{B}) \times 100 \%$. "A" refers to the concentration of the peak of the transcript with exon 20 spliced out, and " $\mathrm{B}$ " refers to the concentration of the peak of the transcript with exon 20 spliced in.

\section{Results}

\section{Association of the Alternatively Spliced RPGRIP1L}

\section{Isoform with Genotypes}

The SNV rs8050354 in RPGRIP1L was reported by the DLPFC sQTLs data set [23] to be associated with alternative splicing, leading to RPGRIP1L exon 20 retention. This SNV was associated with schizophrenia and bipolar disorder in a combined analysis $(p=4.48 \mathrm{E}-05)$ [24]. Because rs8050354 is in intron 24 of RPGRIP1L and the predicted skipped exon is exon 20, we sought to identify SNVs closer to the skipped exon that would be more likely to be the causal variant for the alternative splicing event by identifying SNV s in high LD with rs8050354. rs7203525 is in intron 19 of RPGRIP1L and is in high LD with rs8050354 $\left(r^{2}=0.83, D^{\prime}=0.96\right)[31]$; therefore, we studied this SNV in relation to RPGRIP1L exon 20 skipping as well as rs8050354.

In 92 genotyped brain tissue samples, PCR cDNA amplification identified two RPGRIP1L transcripts: (1) a 239-bp fragment corresponding to the RPGRIP1L transcript including exon 20 and (2) a 137-bp fragment corresponding to a smaller RPGRIP1L transcript with exon 20 spliced out (Fig. 1a). We found striking variation in the levels of these two RPGRIP1L transcripts between individuals, which appeared by inspection to be correlated with the genotypes of rs8050354 and rs7203525. When these two isoforms were then quantified in each of the brain tissue samples using ddPCR, the RPGRIP1L transcript with exon 20 spliced out was significantly decreased in the DLPFC of individuals with the schizophrenia and bipolar disorder risk alleles rs8050354(T) and rs7203525(T) with a dose allele effect (rs8050354: $p<$ $2 \mathrm{E}-16, F(2,84)=135.9 ;$ rs7203525: $p<2 \mathrm{E}-16, F(2,84)=$ 490.3) (Fig. 1b). The RPGRIP1L transcript with exon 20 spliced out was also significantly decreased in the hippo- 


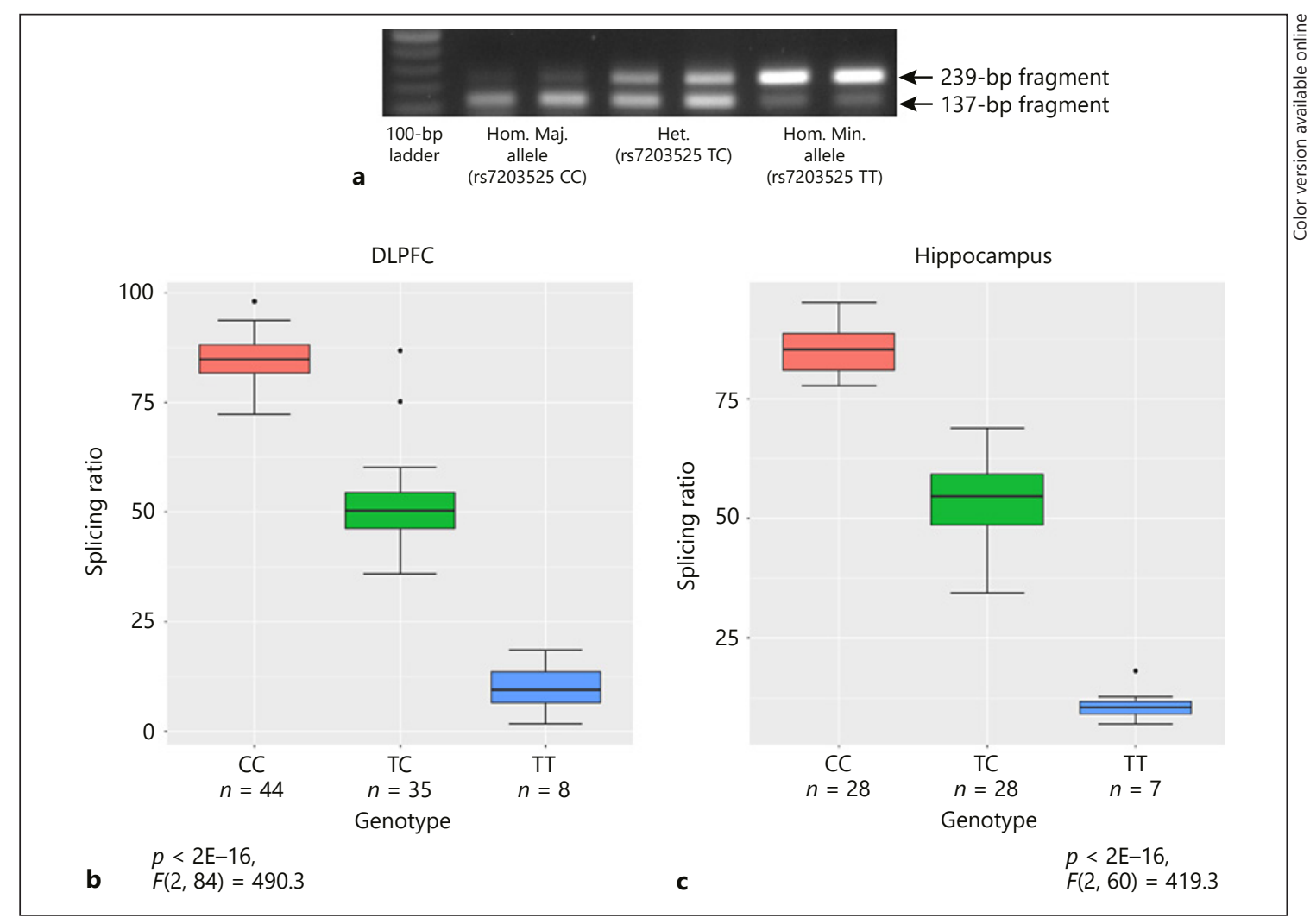

Fig. 1. Schizophrenia and bipolar disorder risk alleles associated with a decrease in RPGRIP1L exon 20-skipped transcript. a cDNA PCR amplification using forward and reverse primers in exons 19 and 21 of $R P G R I P 1 L$, respectively, amplified a 239-bp fragment corresponding to the full-length RPGRIP1L transcript and a 137bp fragment corresponding to a smaller RPGRIP1L transcript isoform excluding exon 20 . b The splicing ratio of the RPGRIP1L exon 20-spliced-out transcript compared to all RPGRIP1L transcripts amplified shows a significant decrease with the schizophre-

campus of individuals with the schizophrenia and bipolar disorder risk alleles rs8050354(T) and rs7203525(T) with a dose allele effect (rs8050354: $p<2 \mathrm{E}-16, F(2,60)=99.61$; rs7203525: $p<2 \mathrm{E}-16, F(2,60)=419.3)$ (Fig. 1c).

\section{rs7203525 Influences Alternative Splicing of RPGRIP1L}

A minigene assay was performed to identify the causal variant of RPGRIP1L exon 20 skipping. The RPGRIP1L region including intron 19 (which contains rs7203525), exon 20, and intron 20 was PCR amplified in two different individuals, one homozygous for the major allele haplotype containing rs7203525(C) and the other homozygous for the minor allele haplotype containing rs7203525(T), and then cloned into the RHCglo vector (Fig. 2a). These plasmids were transfected into the human nia and bipolar disorder risk allele rs7203525(T) in an allele dosedependent manner in the dorsolateral prefrontal cortex (DLPFC). c The splicing ratio of the RPGRIP1L exon 20-spliced-out transcript compared to all RPGRIPIL transcripts amplified shows a significant decrease with the schizophrenia and bipolar disorder risk allele rs7203525(T) in an allele dose-dependent manner in the hippocampus. Hom., homozygous; Het., heterozygous; Maj., major; Min., minor.

neuroblastoma cell line SH-SY5Y, and RNA was extracted $24 \mathrm{~h}$ later to allow for expression. PCR amplification of the cDNA from each of these transfections identified a greater proportion of exon 20 skipping with the major allele haplotype containing rs7203525(C) (Fig. 2b), consistent with the findings from the brain tissue samples.

One additional SNV (rs77511317) is located in the RPGRIP1L region cloned within the minigene construct. To distinguish the causal variant from the rs77511317 variants located on the splicing haplotype, we used sitedirected mutagenesis to change the rs7203525 allele (C) on the major allele haplotype to the minor allele and the minor allele $(\mathrm{T})$ on the minor allele haplotype to the major allele (Fig. 2a). These plasmids were then transfected into SH-SY5Y cells and RNA was extracted. PCR amplification of the cDNA from each of these transfected cells 


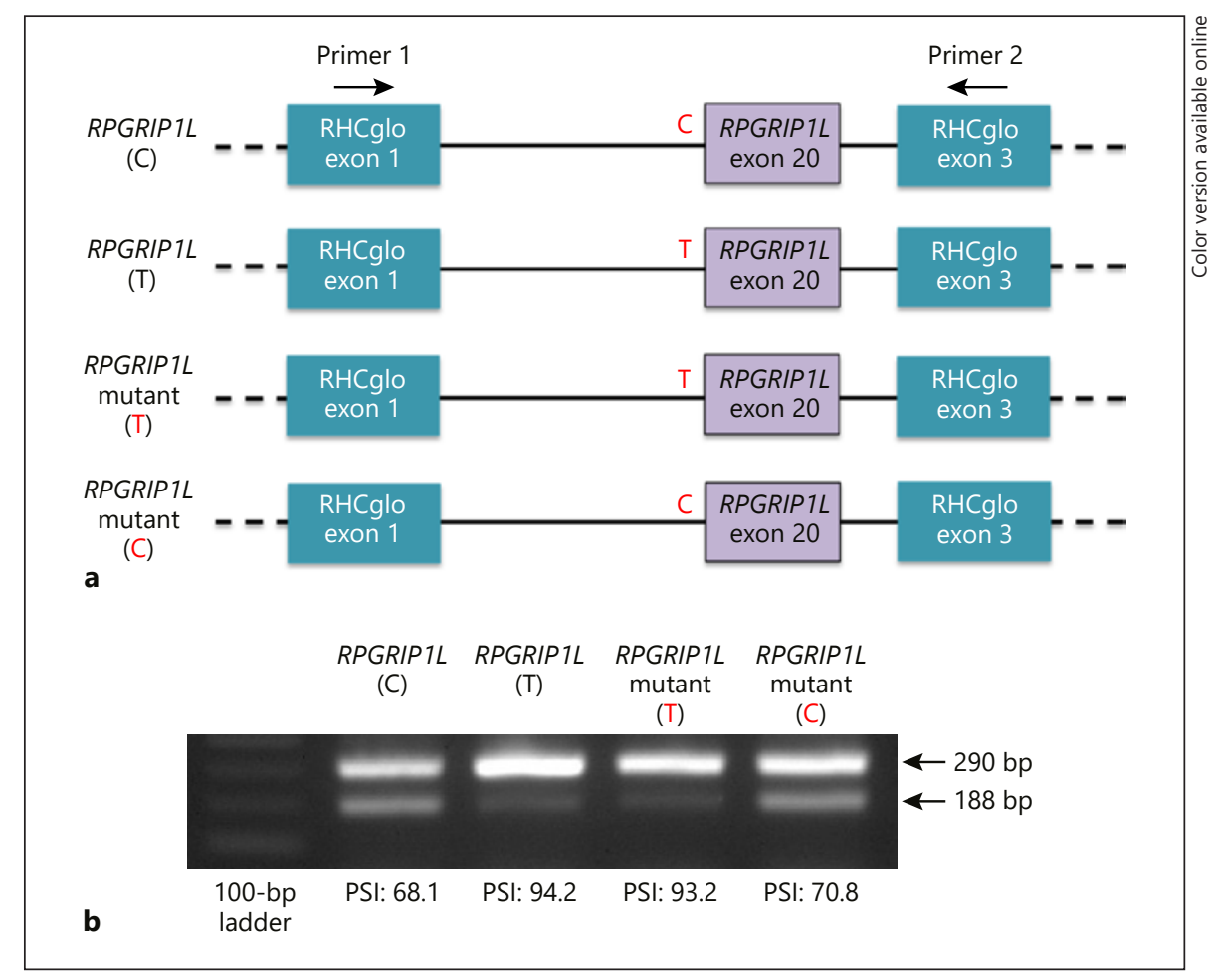

Fig. 2. $R P G R I P 1 L$ variant $\mathrm{rs} 7203525$ influences alternative splicing. a Schematic of RPGRIP1L RHCglo plasmids. The plasmids consist of intron 19, exon 20, and intron 20 of RPGRIP1L cloned in between the two endogenous exons of the RHCglo vector. The RPGRIP1L major allele haplotype contains rs7203525(C), and the RPGRIP1L minor allele haplotype contains rs7203525(T). The RPGRIP1L mutant plasmids had rs7203525 altered through sitedirected mutagenesis such that the RPGRIP1L major allele haplotype mutant contained rs7203525(T) and the RPGRIP1L minor allele haplotype mutant contained rs7203525(C). Dashed lines are indicative of the continuation of the RHCglo plasmid. The forward and reverse primers in the endogenous exons of the RHCglo minigene plasmid used for cDNA amplification are indicated with arrows. b RPGRIP1L variants influence splicing. cDNA from $\mathrm{SH}-$ SY5Y cells transfected with the RPGRIP1L RHCglo plasmids amplified a 290-bp fragment corresponding to the RHCglo exons with $R P G R I P 1 L$ exon 20 spliced in, and a 188-bp fragment corresponding to the RHCglo exons with RPGRIP1L exon 20 spliced out. A higher proportion of the spliced-out fragment was observed with the RPGRIP1L plasmid containing rs7203525(C) regardless of the other single nucleotide variants on the haplotype.

contain exon 20; thus, this variant shifts expression of the common isoform to an alternative form. The full-length RPGRIP1L transcript encodes for a protein with 1,315 amino acids, while the RPGRIP1L transcript skipping exon 20 causes an in-frame deletion of 34 amino acids and results in a protein with 1,281 amino acids. Two sequence motif-based protein prediction methods (InterPro and Pfam) and one automated function prediction method (PredictProtein) were employed to analyze functional differences between the full-length and alternatively spliced RPGRIP1L protein sequences [32-35]. The deletion of 34 amino acids resulting from exon 20 skipping of RPGRIP1L does not affect any known domains identified by InterPro or Pfam. PredictProtein identified two protein-binding regions in the full-length RPGRIP1L protein sequence and four protein-binding regions in 
the exon 20-skipped RPGRIP1L protein sequence. As RPGRIP1L has been found to interact with NEK4 [18], this difference in protein-binding regions between the two isoforms may have implications for NEK4 binding; however, this is unclear from the prediction data. Therefore, the effect on RPGRIP1L protein function from exon 20 skipping of this transcript is currently unknown, and functional studies would be necessary to elucidate the exact biological differences between these two proteins.

The RPGRIP1L protein plays an important role in primary ciliary function, and it contributes to sonic hedgehog signaling as well as ciliary proteasomal and autophagic activity [1, 2, 36, 37]. The developmental and brain malformation phenotypes of Joubert syndrome and Meckel syndrome caused by loss-of-function variants in RPGRIP1L implicate this gene as critical for these processes. As RPGRIP1L is a gene that is necessary for embryonic development and brain formation, the variant identified in this study may affect development as well, and may even influence the Joubert syndrome phenotypes. Further, this variant shows a trend for association with both schizophrenia and bipolar disorder and may contribute to a risk for psychiatric disorders.

The role of the primary cilia has been increasingly indicated in the risk for psychiatric disorders. One of the most thoroughly studied risk genes for psychiatric disorders is disrupted in schizophrenia 1 (DISC1), implicated by a translocation segregating in a large, multiply affected pedigree [38]. DISC1 has been found to localize to, and play a role in, the function of primary cilia [39]. DISC1 localized to the basal bodies of the primary cilia in rat striatal neurons and a fibroblast cell line, and knockdown of DISC1 in these cells resulted in decreased primary cilia, an effect which was restored after reexpression of DISC1 [39]. Interestingly, that study also showed that the dopamine receptors D1R and D2R, both highly implicated in psychiatric disorders, localized to the primary cilia on these cells [39]. A more recent study by Marley and von Zastrow [9] showed that knockdown of 20 genes associated with psychiatric disorders either through interactions with DISC1, rare variants associated with psychiatric disorders, or association by GWAS also resulted in decreased ciliated cells. Interestingly, transfecting these cells with one of the top schizophrenia GWAS-associated genes, MIR137 [13], increased ciliation and the length of the cilia [9]. Furthermore, lithium, the most common and effective treatment of bipolar disorder, has also been found to increase the length of primary cilia in striatal neuronal cell culture and in the mouse brain [40]. Thus, multiple lines of evidence implicate the primary cilia in psychiatric disorders, and a shift to this RPGRIP1L isoform in the brain may also alter primary ciliary function.

Interestingly, RPGRIP1L knockout murine embryonic fibroblasts have decreased ciliary proteasome activity, and treatment with sulforaphane leads to a recovery of the ciliary proteasome activity $[2,37]$. Sulforaphane, found in cruciform plants, particularly in broccoli sprouts, has garnered interest from clinical trials for cancer, diabetes, schizophrenia, and autism [41-45]. Of particular interest, sulforaphane has been found in mice to prevent cognitive deficits induced by phencyclidine, an NMDAR antagonist that mimics symptoms of schizophrenia and has also been reported to cause impaired primary cilia and altered levels of RPGRIP1L mRNA in rat and mouse brains [11]. This provides an interesting avenue of future study with regard to the potential contribution of RPGRIP1L and dysfunctional primary cilia to psychiatric disease.

Also relevant to psychiatric disorders and brain function, RPGRIP1L has also been found to be important for left-right asymmetry development [1] - implicated in schizophrenia by decades of research including, more recently, structural and functional imaging [46, 47]. Oertel et al. [48] identified reduced left compared to right asymmetry in the temporal lobe of schizophrenia patients when compared to controls based on gray matter volume and functional activation. First-degree unaffected relatives of schizophrenia patients were also studied, and they showed an intermediate reduced-asymmetry phenotype, suggesting a genetic component. Thus, the role of this gene and alternative isoforms are interesting candidates for study of variation in left-right brain asymmetry and laterality.

In summary, we identified an SNV, rs7203525, that influences an alternative splicing event in RPGRIP1L, leading to altered ratios of isoforms with exon 20 retention. As RPGRIP1L is an important developmental gene, this identification paves the way for future studies to unravel the altered protein function of these two RPGRIP1L isoforms and determine how they contribute to embryonic and neural processes, as well as to the RPGRIP1L disorders Joubert syndrome and Meckel syndrome.

\section{Acknowledgements}

The brain tissue samples were obtained from the Douglas-Bell Canada Brain Bank and the NIH NeuroBioBank (Harvard Brain Tissue Resource Center and Eunice Kennedy Shriver National Institute of Child Health and Human Development). Funding was provided by grants from the Hospital for Sick Children Psychiatric Endowment Fund and the Canadian Institutes of Health Research. E.R. was supported by a Restracomp Studentship from the Research Training Centre of the Hospital for Sick Children. 


\section{Statement of Ethics}

The study was approved by the Hospital for Sick Children and University Health Network Research Ethics Boards.

\section{Disclosure Statement}

The authors declare no conflicts of interest.

\section{References}

1 Vierkotten J, Dildrop R, Peters T, Wang B, Rüther U. Ftm is a novel basal body protein of cilia involved in Shh signalling. Development. 2007 Jul;134(14):2569-77.

2 Wiegering A, Rüther U, Gerhardt C. The ciliary protein Rpgripll in development and disease. Dev Biol. 2018 Oct;442(1):60-8.

3 Delous M, Baala L, Salomon R, Laclef C, Vierkotten J, Tory $\mathrm{K}$, et al. The ciliary gene RPGRIP1L is mutated in cerebello-oculo-renal syndrome (Joubert syndrome type B) and Meckel syndrome. Nat Genet. 2007 Jul;39(7): 875-81.

4 Doherty D. Joubert syndrome: insights into brain development, cilium biology, and complex disease. Semin Pediatr Neurol. 2009 Sep; 16(3):143-54

5 Bryant L, Lozynska O, Maguire AM, Aleman TS, Bennett J. Prescreening whole exome sequencing results from patients with retinal degeneration for variants in genes associated with retinal degeneration. Clin Ophthalmol. 2017 Dec;12:49-63.

6 Khanna H, Davis EE, Murga-Zamalloa CA, Estrada-Cuzcano A, Lopez I, den Hollander $\mathrm{AI}$, et al. A common allele in RPGRIP1L is a modifier of retinal degeneration in ciliopathies. Nat Genet. 2009 Jun;41(6):739-45.

7 Guo J, Higginbotham H, Li J, Nichols J, Hirt J, Ghukasyan V, et al. Developmental disruptions underlying brain abnormalities in ciliopathies. Nat Commun. 2015 Jul;6(1):7857.

8 Guemez-Gamboa A, Coufal NG, Gleeson JG. Primary cilia in the developing and mature brain. Neuron. 2014 May;82(3):511-21.

9 Marley A, von Zastrow M. A simple cell-based assay reveals that diverse neuropsychiatric risk genes converge on primary cilia. PLoS One. 2012;7(10):e46647.

10 Pruski M, Lang B. Primary Cilia - An Underexplored Topic in Major Mental Illness. Front Psychiatry. 2019 Mar;10:104.

11 Shiwaku H, Umino A, Umino M, Nishikawa T. Phencyclidine-induced dysregulation of primary cilia in the rodent brain. Brain Res. 2017 Nov; 1674:62-9.

12 O’Donovan MC, Craddock N, Norton N, Williams H, Peirce T, Moskvina V, et al.; Molecular Genetics of Schizophrenia Collaboration. Identification of loci associated with schizophrenia by genome-wide association and follow-up. Nat Genet. 2008 Sep;40(9): 1053-5.

13 Schizophrenia Working Group of the Psychiatric Genomics Consortium. Biological insights from 108 schizophrenia-associated genetic loci. Nature. 2014 Jul;511(7510):421-7.
14 Jiang Y, Zhang H. Propensity score-based nonparametric test revealing genetic variants underlying bipolar disorder. Genet Epidemiol. 2011 Feb;35(2):125-32.

15 Goes FS, Pirooznia M, Parla JS, Kramer M, Ghiban E, Mavruk S, et al. Exome Sequencing of Familial Bipolar Disorder. JAMA Psychiatry. 2016 Jun;73(6):590-7.

16 Iossifov I, O’Roak BJ, Sanders SJ, Ronemus M, Krumm N, Levy D, et al. The contribution of de novo coding mutations to autism spectrum disorder. Nature. 2014 Nov;515(7526):216-21.

17 Summers AC, Snow J, Wiggs E, Liu AG, Toro C, Poretti A, et al.; NISC Comparative Sequencing Program. Neuropsychological phenotypes of 76 individuals with Joubert syndrome evaluated at a single center. Am J Med Genet A. 2017 Jul;173(7):1796-812.

18 Coene KL, Mans DA, Boldt K, Gloeckner CJ, van Reeuwijk J, Bolat E, et al. The ciliopathyassociated protein homologs RPGRIP1 and RPGRIP1L are linked to cilium integrity through interaction with Nek4 serine/threonine kinase. Hum Mol Genet. 2011 Sep; 20(18):3592-605.

19 Gusev A, Mancuso N, Won H, Kousi M, Finucane HK, Reshef Y, et al.; Schizophrenia Working Group of the Psychiatric Genomics Consortium. Transcriptome-wide association study of schizophrenia and chromatin activity yields mechanistic disease insights. Nat Genet. 2018 Apr;50(4):538-48.

20 Reble E, Dineen A, Barr CL. The contribution of alternative splicing to genetic risk for psychiatric disorders. Genes Brain Behav. 2018 Mar;17(3):e12430.

21 Xiong HY, Alipanahi B, Lee LJ, Bretschneider $\mathrm{H}$, Merico D, Yuen RK, et al. RNA splicing. The human splicing code reveals new insights into the genetic determinants of disease. Science. 2015 Jan;347(6218):1254806.

22 Ardlie KG, Deluca DS, Segre AV, Sullivan TJ, Young TR, Gelfand ET, et al.; GTEx Consortium. Human genomics. The Genotype-Tissue Expression (GTEx) pilot analysis: multitissue gene regulation in humans. Science. 2015 May;348(6235):648-60.

23 Takata A, Matsumoto N, Kato T. Genomewide identification of splicing QTLs in the human brain and their enrichment among schizophrenia-associated loci. Nat Commun. 2017 Feb;8(1):14519.

24 Bipolar Disorder and Schizophrenia Working Group of the Psychiatric Genomics Consortium. Genomic Dissection of Bipolar Disorder and Schizophrenia, Including 28 Subphenotypes. Cell. 2018 Jun;173(7):1705-15.e16.
25 Ongen H, Dermitzakis ET. Alternative Splicing QTLs in European and African Populations. Am J Hum Genet. 2015 Oct;97(4):567-75.

26 Monlong J, Calvo M, Ferreira PG, Guigó R. Identification of genetic variants associated with alternative splicing using sQTLseekeR. Nat Commun. 2014 Aug;5(1):4698.

27 Rivas MA, Pirinen M, Conrad DF, Lek M, Tsang EK, Karczewski KJ, et al.; GTEx Consortium; Geuvadis Consortium. Human genomics. Effect of predicted protein-truncating genetic variants on the human transcriptome. Science. 2015 May;348(6235):666-9.

28 Purcell S, Neale B, Todd-Brown K, Thomas L, Ferreira MA, Bender D, et al. PLINK: a tool set for whole-genome association and population-based linkage analyses. Am J Hum Genet. 2007 Sep;81(3):559-75.

29 Das S, Forer L, Schönherr S, Sidore C, Locke AE, Kwong A, et al. Next-generation genotype imputation service and methods. Nat Genet. 2016 Oct;48(10):1284-7.

30 Singh G, Cooper TA. Minigene reporter for identification and analysis of cis elements and trans factors affecting pre-mRNA splicing. Biotechniques. 2006 Aug;41(2):177-81.

31 Ward LD, Kellis M. HaploReg: a resource for exploring chromatin states, conservation, and regulatory motif alterations within sets of genetically linked variants. Nucleic Acids Res. 2012 Jan;40(Database issue):D930-4.

32 Mitchell AL, Attwood TK, Babbitt PC, Blum M, Bork P, Bridge A, et al. InterPro in 2019: improving coverage, classification and access to protein sequence annotations. Nucleic Acids Res. 2019 Jan;47(D1):D351-60.

33 El-Gebali S, Mistry J, Bateman A, Eddy SR, Luciani A, Potter SC, et al. The Pfam protein families database in 2019. Nucleic Acids Res. 2019 Jan;47(D1):D427-32.

34 Finn RD, Coggill P, Eberhardt RY, Eddy SR, Mistry J, Mitchell AL, et al. The Pfam protein families database: towards a more sustainable future. Nucleic Acids Res. 2016 Jan; 44(D1):D279-85.

35 Yachdav G, Kloppmann E, Kajan L, Hecht M, Goldberg T, Hamp T, et al. PredictProtein an open resource for online prediction of protein structural and functional features. Nucleic Acids Res. 2014 Jul;42(Web Server issue):W337-43.

36 Struchtrup A, Wiegering A, Stork B, Rüther U, Gerhardt C. The ciliary protein RPGRIP1L governs autophagy independently of its proteasome-regulating function at the ciliary base in mouse embryonic fibroblasts. Autophagy. 2018;14(4):567-83. 
37 Gerhardt C, Lier JM, Burmühl S, Struchtrup A, Deutschmann K, Vetter M, et al. The transition zone protein Rpgrip1l regulates proteasomal activity at the primary cilium. J Cell Biol. 2015 Jul;210(1):115-33.

38 Bradshaw NJ, Porteous DJ. DISC1-binding proteins in neural development, signalling and schizophrenia. Neuropharmacology. 2012 Mar;62(3):1230-41.

39 Marley A, von Zastrow M. DISC1 regulates primary cilia that display specific dopamine receptors. PLoS One. 2010 May;5(5):e10902.

40 Miyoshi K, Kasahara K, Miyazaki I, Asanuma M. Lithium treatment elongates primary cilia in the mouse brain and in cultured cells. Biochem Biophys Res Commun. 2009 Oct; 388(4):757-62.
41 Bayat Mokhtari R, Baluch N, Homayouni TS, Morgatskaya E, Kumar S, Kazemi P, et al. The role of sulforaphane in cancer chemoprevention and health benefits: a mini-review. J Cell Commun Signal. 2018 Mar;12(1):91-101.

42 Huang $\mathrm{C}, \mathrm{Wu}$ J, Chen D, Jin J, Wu Y, Chen Z. Effects of sulforaphane in the central nervous system. Eur J Pharmacol. 2019 Jun;853:15368.

43 Bent S, Lawton B, Warren T, Widjaja F, Dang K, Fahey JW, et al. Identification of urinary metabolites that correlate with clinical improvements in children with autism treated with sulforaphane from broccoli. Mol Autism. 2018 May;9(1):35.

44 Sedlak TW, Nucifora LG, Koga M, Shaffer LS, Higgs C, Tanaka T, et al. Sulforaphane Augments Glutathione and Influences Brain Metabolites in Human Subjects: A Clinical Pilot Study. Mol Neuropsychiatry. 2018 May;3(4): 214-22.
45 Sedlak TW, Paul BD, Parker GM, Hester LD, Snowman AM, Taniguchi Y, et al. The glutathione cycle shapes synaptic glutamate activity. Proc Natl Acad Sci USA. 2019 Feb;116(7): 2701-6.

46 Ribolsi M, Daskalakis ZJ, Siracusano A, Koch G. Abnormal asymmetry of brain connectivity in schizophrenia. Front Hum Neurosci. 2014 Dec;8:1010.

47 Mitchell RL, Crow TJ. Right hemisphere language functions and schizophrenia: the forgotten hemisphere? Brain. 2005 May; 128(Pt 5):963-78.

48 Oertel V, Knöchel C, Rotarska-Jagiela A, Schönmeyer R, Lindner M, van de Ven V, et al. Reduced laterality as a trait marker of schizophrenia - evidence from structural and functional neuroimaging. J Neurosci. 2010 Feb;30(6):2289-99. 